\title{
NRSSD: Normalizing Received Signal Strength to Address Device Diversity Problem in Fingerprinting Positioning
}

\author{
Chunxiu Li ${ }^{\mathrm{a}}$, Jianli Zhao ${ }^{\mathrm{a},{ }^{*}}$, Qiuxia Sun ${ }^{\mathrm{b}}$, Xiang Gao ${ }^{\mathrm{a}}$, Guoqiang Sun ${ }^{\mathrm{a}}$, and Chendi Zhu ${ }^{\mathrm{a}}$ \\ ${ }^{a}$ School of Computer Science and Engineering, Shandong University of Science and Technology, Qingdao, 266000, China \\ ${ }^{b}$ School of Mathematics and Systems Science, Shandong University of Science and Technology, Qingdao, 266000, China
}

\begin{abstract}
The WiFi-based fingerprinting technique is widely adopted for indoor positioning due to its cost-effectiveness compared to other infrastructure-based positioning methods. However, the WiFi-based technique still faces the problem of device diversity in the application of an indoor positioning system. Previous studies have faced two main challenges. One is the curse of computational dimensionality in online positioning, while the other is the issue of low positioning accuracy in real applications. In this paper, we propose to normalize the observable Access Point (AP) signal strength to eliminate the influence of device diversity and avoid a dimension disaster. Experimental results show that our algorithm based on the normalization Received Signals Strength (RSS) not only solves the problem of device diversity but also outperforms three other baseline methods.
\end{abstract}

Keywords: fingerprinting positioning; device diversity; normalization; positioning accuracy

(Submitted on November 10, 2018; Revised on December 13, 2018; Accepted on January 14, 2019)

(C) 2019 Totem Publisher, Inc. All rights reserved.

\section{Introduction}

With the rapid development of mobile technology and the promotion of wireless communication networks, the demands for Location Based Services (LBS) have increased dramatically. The limitations of people's special daily living habits and the deficiency of mature outdoor positioning techniques in facing indoor environments promote the research of indoor positioning techniques [1]. Among them, the WiFi-based indoor fingerprinting positioning technique stands out due to its low cost of development, high positioning accuracy, and wide range of application, and it has gradually become the focus of research in the field of indoor positioning techniques [2]. The fingerprint-based positioning method is usually conducted in two phases: an offline phase followed by an online phase [3]. In the offline phase, a site survey is executed to collect the RSS of all the received signals from different APs at known positions throughout the indoor area. These known positions are Reference Points (RPs). All RSS fingerprints and their corresponding RPs are stored in a database, which is called the radio map. In the online phase, a user measures an RSS fingerprint at an unknown position, which is called the querying fingerprint. Then, the querying fingerprint is compared with the RSS fingerprints stored in the radio map, and the estimated position is computed using different algorithms (e.g., the k-Nearest Neighbor (KNN) and the weighted k-Nearest Neighbor (WKNN)) [4]. However, the technique still faces some challenges in the application of the indoor positioning system (IPS). Device diversity is one such challenge for fingerprinting. The traditional fingerprint positioning method requires the training devices (i.e., the device used to build the radio map during the offline phase) and testing devices (i.e., the device used for positioning during the online phase) to be consistent. However, real applications of IPS will bring diverse devices, and the RSS variation due to the device diversity will decrease the positioning accuracy severely.

In this paper, we design an indoor fingerprinting positioning algorithm based on the normalizing RSS, which is called the NRSSD. The rest of the paper is organized as follows: Section 2 discusses related works in the literature. Section 3 analyzes the influence of device diversity in the fingerprinting positioning technique and introduces the details of the NRSSD algorithm. We evaluate our algorithm in Section 4. Finally, we conclude our work in Section 5.

* Corresponding author.

E-mail address: zhaojianli@gmail.com 


\section{Related Works}

To solve the problem of device diversity, which hinders the application of IPS, many scholars have proposed different solutions from different aspects. A. W. Tsui. focused on the influencing factors of device diversity in indoor positioning techniques [5-6]. These factors include wireless chips, antennas, and operating systems. J. G. Park. pointed out that different devices in the same position usually acquire different RSS measurements [7]; however, the traditional Fingerprinting Positioning algorithm regards these RSS measurements as different RSS fingerprints, and so it decreases positioning accuracy.

In recent years, there have been two research directions to resolve the problem of device diversity: offline correction and online correction. The offline correction method adopts transformation functions to train the relevant regression coefficients between the training devices and testing devices [8-12]. However, this method requires that the heterogeneous devices must be known and available in advance such that the regression procedure can be executed to derive the pair-wise linear relationships. This requirement imposes restrictions on wide applications with a mass of new and unknown devices, because it is unrealistic to calibrate RSS for every new testing device. The most popular online correction methods are the DIFF algorithm [13], the HLF algorithm [14-15], and the SSD algorithm [16]. The DIFF algorithm takes the differences between all possible AP pairs as the differential fingerprints; however, it dramatically increases the dimensionality of the fingerprints, especially in indoor areas covered by a large number of APs, thus leading to higher computational complexity compared to the traditional RSS fingerprints. To address this issue, the SSD algorithm creates the fingerprints by subtracting the reference AP RSS measurement from the RSS measurements of the other APs. However, selecting the right reference AP is critical. Using an unobservable AP as a reference AP will cause poor positioning accuracy, especially in a large-scale scenario where APs do not provide ubiquitous coverage; therefore, the unobservable AP inevitably is selected as the reference AP (Section 3 and 4 introduce it in detail). Here, the unobservable AP represents the AP where no signal is received at a certain RP; traditionally, it is set to a small constant value (such as $-95 \mathrm{dBm}$ ). The HLF algorithm combines the normalized logarithm ratios of the RSS power from different APs to remove the device-dependent component. However, the resulting RSS logarithm ratios do not totally mitigate the hardware variations [16].

The main purpose of this paper is to solve the problem of device diversity. We present the indoor fingerprinting positioning algorithm based on the normalization RSS. The main contributions of this paper are as follows.

We design an indoor fingerprinting algorithm based on the normalizing RSS (NRSSD) to address device diversity. This algorithm does not require one to train the mapping model among heterogeneous devices in advance and only uses the RSS to eliminate the influencing factors of device diversity. Moreover, the NRSSD algorithm decreases the influence of unobservable AP on device diversity processing.

\section{Normalizing Received Signal Strength to Address Device Diversity in Fingerprinting Positioning}

The fingerprinting positioning algorithm emphasizes the consistency of training devices and testing devices. However, it is unrealistic to require all users to use the same devices, which seriously hinders the popularization of the fingerprinting positioning technique. To solve the problem of device diversity, this paper conducts theoretical research and experiments on the influential factors of device diversity. Then, we design the NRSSD. Table 1 summarizes the symbols used for the remainder of this paper.

Table 1. Table of the symbols used in this paper

\begin{tabular}{|c|c|}
\hline Symbol & Definition \\
\hline $\mathbf{M}$ & Number of APs $\mathbf{A P}=\left\{A P_{1}, A P_{2}, \cdots, A P_{M}\right\}$ \\
\hline $\mathbf{N}$ & Number of RPs $\mathbf{R P}=\left\{R P_{1}, R P_{2}, \cdots, R P_{N}\right\}$ \\
\hline $\mathbf{R S S}_{i, .}$ & RSS vector at the $R P_{i}$ for all APs \\
\hline $\mathbf{R S S}_{q}$ & RSS vector at the unknown position $q$ for all APs \\
\hline$\left(\hat{x}_{q}, \hat{y}_{q}\right)$ & Estimated position for $q$ \\
\hline
\end{tabular}

\subsection{Analysis of the Influence of Diverse Devices for the Fingerprinting Positioning Algorithm}

To show the influence of device diversity on the indoor fingerprinting positioning algorithm, we conducted some experiments. We used the KIOS dataset (Section 4 describes the details of the KIOS dataset [10]) as the testing source. First, we randomly selected four RPs and compared the RSS for the nine APs from each of the four kinds of devices at each of the four RPs. Next, we conducted cross-positioning experiments that used one device's training fingerprints to locate the testing 
fingerprints of the four other devices. Finally, Figure 1 shows the RSS from the diverse devices at the same position, and Table 2 shows the average positioning errors of the cross-positioning experiments between diverse devices.

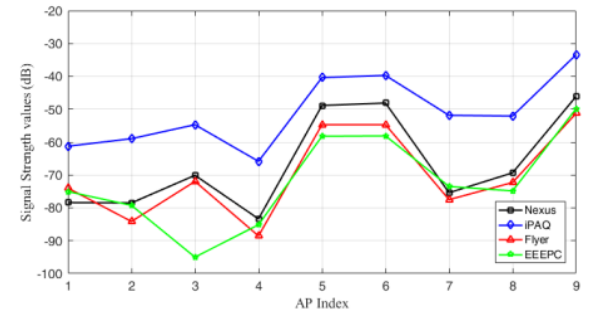

(a) RP1

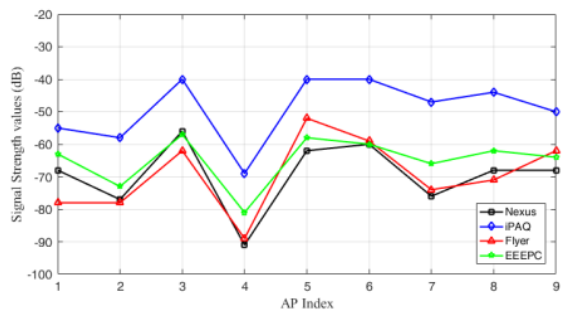

(c) RP3

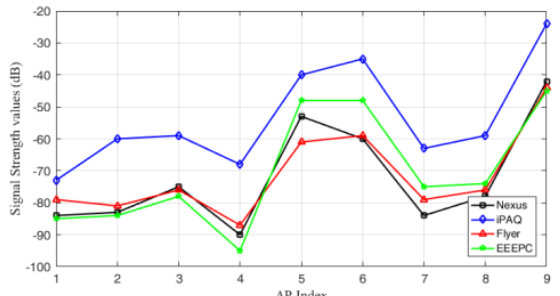

(b) RP2

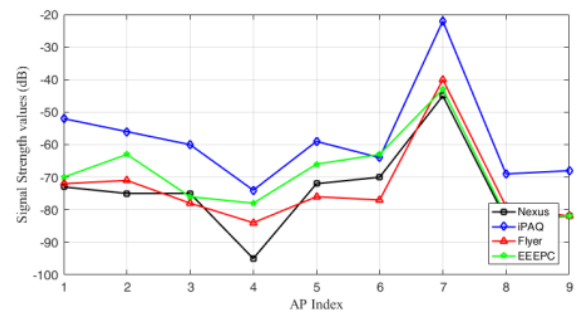

(d) RP4

Figure 1. Comparison of the RSS from diverse devices at the same position

Figure 1 shows the numerical comparison of the signal values from nine APs acquired by four devices at four positions. Even in the same position, the RSS values for the same AP from the four kinds of devices are different, and the differences lead to the radio map from one device not being suitable for the online positioning of the other devices. Next, we use Figure 1(a) as an example to make a comparison.

Table 2. Comparison of the average positioning errors between diverse devices

\begin{tabular}{|c|c|c|c|c|c|}
\hline \multicolumn{2}{|c|}{ Average positioning Errors (m) } & \multicolumn{4}{|c|}{ Testing devices } \\
\cline { 2 - 5 } & Nexus & Nexus & IPAQ & Flyer & EEEPC \\
\hline \multirow{3}{*}{$\begin{array}{l}\text { Training } \\
\text { Devices }\end{array}$} & IPAQ & 7.2752 & 6.2263 & 2.8005 & 2.8733 \\
\cline { 2 - 5 } & Flyer & 2.7189 & 2.7124 & 7.0854 & 6.2458 \\
\cline { 2 - 6 } & EEEPC & 2.9959 & 4.8411 & 1.9113 & 3.0563 \\
\cline { 2 - 5 } & & & 4.3880 & 3.0309 & 2.2528 \\
\hline
\end{tabular}

Table 2 shows that when the training and testing devices are the same type, the positioning errors are lower than those of the diverse devices. Therefore, we can infer that the difference in the signal values for the same AP among diverse devices affects the positioning accuracy.

In theory, this paper analyzes the reason for the differences in the RSS values acquired by diverse devices at the same position for the same AP. We briefly describe the device's dependency and related factors. Equation (1) represents the RSS calculation method [2].

$$
P_{i, j}(d)=10 \log _{10}\left(\frac{P_{i} G_{i} \lambda_{i}^{2} G_{j}}{16 \pi^{2} d_{0}^{2} L_{d}}\right)-10 \beta \log _{10}\left(\frac{d}{d_{0}}\right)+\xi
$$

Where $P_{i, j}(d)$ is the RSS from a given $A P_{i}$ at distance $d$ for the device $j, P_{i}$ is the $A P_{i}$ transmitted power, $G_{i}$ and $\lambda_{i}$ are the $A P_{i}$ antenna gain and the transmitted carrier's wavelength, respectively, $G_{j}$ is the device $j$ 's antenna gain, $d_{0}$ is a reference distance that is usually $d_{0}=1 \mathrm{~m}, L_{d}$ is the system loss factor, $\beta$ is the path loss exponent, and the noise is $\zeta \sim N\left(0, \sigma^{2}\right)$. In Equation (1), there are different $G_{j}$ and signal values $P_{i, j}(d)$.

\subsection{Indoor Fingerprinting Positioning Algorithm based on Normalizing RSS}

The previous section showed that the fingerprinting positioning algorithm does not perform well if the problem of diverse devices cannot be solved. Therefore, we design an algorithm based on the normalizing RSS for device diversity, and the following provides the details of the proposed algorithm. 


\subsubsection{The Difference Between RSSs}

Table 2 shows that when the training and testing devices are the same type, the positioning errors are lower than those of the diverse devices. Therefore, we can infer that the difference in the signal values for the same AP among diverse devices affects the positioning accuracy.

We suppose the following simulated situation to analyze the differences between the RSSs in Figure 2. There are two access points in the indoor area, $A P_{1}$ and $A P_{2}$, and one person simultaneously carries device A and device $\mathrm{B}$ to collect the signal strength at a fixed reference point. According to Equation (1), the RSS values acquired by the two devices for the two APs are at the positions shown in Equations (2) and (3).

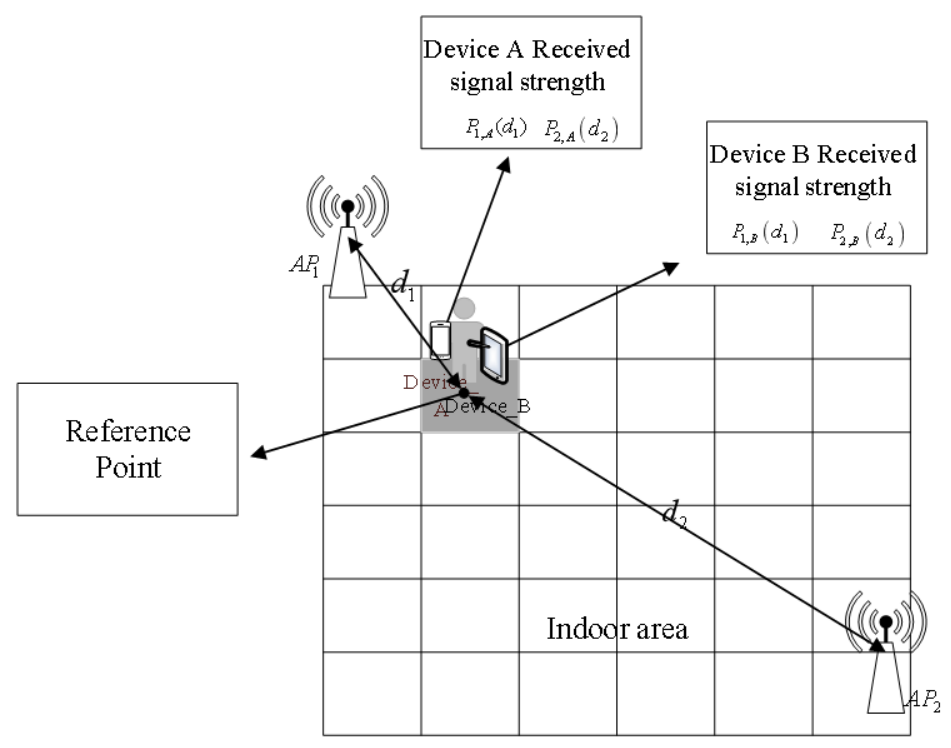

Figure 2. Diagram of the simulated situation

$$
\begin{aligned}
& \left\{\begin{array}{l}
P_{1, A}\left(d_{1}\right)=10 \log _{10}\left(\frac{P_{1} G_{1} \lambda_{1}^{2} G_{A}}{16 \pi^{2} d_{0}^{2} L_{d_{1}}}\right)-10 \beta_{1} \log _{10}\left(\frac{d_{1}}{d_{0}}\right)+\xi_{1} \\
P_{2, A}\left(d_{2}\right)=10 \log _{10}\left(\frac{P_{2} G_{2} \lambda_{2}^{2} G_{A}}{16 \pi^{2} d_{0}^{2} L_{d_{2}}}\right)-10 \beta_{2} \log _{10}\left(\frac{d_{2}}{d_{0}}\right)+\xi_{2}
\end{array}\right. \\
& \left\{\begin{array}{l}
P_{1, B}\left(d_{1}\right)=10 \log _{10}\left(\frac{P_{1} G_{1} \lambda_{1}^{2} G_{B}}{16 \pi^{2} d_{0}^{2} L_{d_{1}}}\right)-10 \beta_{1} \log _{10}\left(\frac{d_{1}}{d_{0}}\right)+\xi_{1} \\
P_{2, B}\left(d_{2}\right)=10 \log _{10}\left(\frac{P_{2} G_{2} \lambda_{2}^{2} G_{B}}{16 \pi^{2} d_{0}^{2} L_{d_{2}}}\right)-10 \beta_{2} \log _{10}\left(\frac{d_{2}}{d_{0}}\right)+\xi_{2}
\end{array}\right.
\end{aligned}
$$

The difference between $G_{A}$ and $G_{B}$ results in the difference between $P_{1, A}\left(d_{1}\right)$ and $P_{1, B}\left(d_{1}\right)$ as well as the difference between $P_{2, A}\left(d_{2}\right)$ and $P_{2, B}\left(d_{2}\right)$. Therefore, we subtract $P_{2, A}\left(d_{2}\right)$ from $P_{1, A}\left(d_{1}\right)$ in Equation (2) to obtain the differential fingerprint $\Delta P_{A}$ and subtract $P_{2, B}\left(d_{2}\right)$ from $P_{1, B}\left(d_{1}\right)$ in Equation (3) to obtain the differential fingerprint $\Delta P_{B}$.

$$
\Delta P_{A}=10 \log _{10}\left(\frac{P_{1} G_{1} \lambda_{1}^{2} L_{d_{2}}}{P_{2} G_{2} \lambda_{2}^{2} L_{d_{1}}}\right)-10 \beta_{1} \log _{10}\left(\frac{d_{1}}{d_{0}}\right)+10 \beta_{2} \log _{10}\left(\frac{d_{2}}{d_{0}}\right)+\left(\xi_{1}-\xi_{2}\right)
$$




$$
\Delta P_{B}=10 \log _{10}\left(\frac{P_{1} G_{1} \lambda_{1}^{2} L_{d_{2}}}{P_{2} G_{2} \lambda_{2}^{2} L_{d_{1}}}\right)-10 \beta_{1} \log _{10}\left(\frac{d_{1}}{d_{0}}\right)+10 \beta_{2} \log _{10}\left(\frac{d_{2}}{d_{0}}\right)+\left(\xi_{1}-\xi_{2}\right)
$$

In Equations (4) and (5), $\Delta P_{A}$ and $\Delta P_{B}$ are the same when the random noise variable $\left(\zeta_{1}-\zeta_{2}\right)$ is removed. Therefore, we use the differential fingerprints $\Delta P_{A}$ and $\Delta P_{B}$ to replace the original RSS since it can effectively eliminate the influence of device antennas' gain factor.

\subsubsection{Description of Normalizing RSS}

The normalized method is a good way to improve the accuracy of some distance algorithms, such as the KNN/WKNN. According to the conclusions given in [17], normalization makes the characteristics among different dimensions reach the same magnitude and greatly improves the accuracy of the distance algorithm. We use the WKNN to estimate the position in the online phase of the fingerprint-based positioning method. Therefore, this paper expounds on the Min-Max linear normalized method to eliminate the influence of device diversity and improve positioning accuracy. First, we subtract the RSS minimum from the original RSS to eliminate the influence of the device antennas' gain factor. Then, we normalize the differential fingerprints to improve the positioning accuracy and reduce the curse of dimensionality compared with other algorithms.

The fundamental principle of the method is that we suppose a numeric attribute $A$, which has $n$ observations $a_{1}, a_{2}, \cdots, a_{n}$. The maximum, $\max _{A}$, and the minimum, $\min _{A}$, of $A$ are used to normalize these observations, and the original data will be transformed linearly by calculating Equation (6).

$$
a_{i}^{\prime}=\frac{a_{i}-\min _{A}}{\max _{A}-\min _{A}}, \quad 1 \leq i \leq n
$$

Suppose that $\boldsymbol{\alpha}=\left[R S S_{1}, R S S_{2}, \cdots, R S S_{M}\right]$ represents the RSS vector at an arbitrary RP from one device for $M$ number of APs. Here, we use the Min-Max linear normalized method to preprocess $\boldsymbol{\alpha}$ and find the maximum of $R S S_{\max }=\left\{R S S_{i}, i=1, \cdots, M\right\}_{\max }$ and the minimum of $R S S_{\min }=\left\{R S S_{i}, R S S_{i} \neq-95, i=1, \cdots, M\right\}_{\min }$. According to (6), we get the numerical value of each vector $\boldsymbol{\alpha}$ mapped to[0,1], and a new vector $\boldsymbol{\alpha}^{\prime}$ is shown in Equation (7).

$$
\boldsymbol{\alpha}^{\prime}=\left[\frac{R S S_{1}-R S S_{\min }}{R S S_{\text {max }}-R S S_{\text {min }}} \mathbf{I}\left(R S S_{1} \neq-95\right), \frac{R S S_{2}-R S S_{\text {min }}}{R S S_{\text {max }}-R S S_{\text {min }}} \mathbf{I}\left(R S S_{2} \neq-95\right), \cdots, \frac{R S S_{M}-R S S_{\text {min }}}{R S S_{\text {max }}-R S S_{\text {min }}} \mathbf{I}\left(R S S_{M} \neq-95\right)\right]
$$

In this formula, I(.) is the indicator function, which is equal to 1 if its argument is true, and it is equal to 0 if its argument is false, which will decrease the influence of unobservable AP for the normalization processing on $\boldsymbol{\alpha}$. Based on Equations (4) and (5), the normalized $\boldsymbol{\alpha}^{\prime}$ has removed the antenna gain factor $G$, and new vector $\boldsymbol{\alpha}^{\prime}$ is shown in Figure 3.

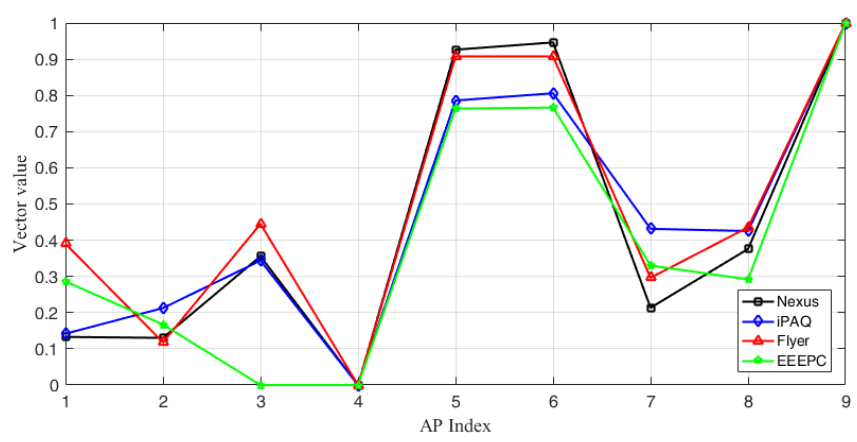

Figure 3. Comparison of the results of the normalized RSS $\boldsymbol{\alpha}^{\prime}$ from different devices

Figure 3 shows the comparison of the normalized RSS for the same AP from the four devices at the same position. 
Compared with Figure 1(a), Figure 3 shows that when four kinds of devices are diverse, the differences in values among the diverse devices are significantly reduced.

Next, we do not consider the influence of the unobservable AP for the normalized RSS and let $R S S_{\text {min }}=\boldsymbol{\alpha}_{\text {min }}$ and $R S S_{\max }=\boldsymbol{\alpha}_{\max }$. We call this method MM. The new normalized vector $\boldsymbol{\alpha}_{1}^{\prime}$ is shown in Equation (8).

$$
\boldsymbol{\alpha}_{1}^{\prime}=\left[\frac{R S S_{1}-R S S_{\text {min }}}{R S S_{\text {max }}-R S S_{\text {min }}}, \frac{R S S_{2}-R S S_{\text {min }}}{R S S_{\text {max }}-R S S_{\text {min }}}, \cdots, \frac{R S S_{M}-R S S_{\text {min }}}{R S S_{\text {max }}-R S S_{\text {min }}}\right]
$$

Figure 4 shows the comparison of the new normalized RSS $\boldsymbol{\alpha}_{1}^{\prime}$ for the same AP from four devices at the same position. Compared with Figure 3, when the four devices are diverse, the differences in the values among the diverse devices are significantly increased, such as $A P_{2}$ and $A P_{4}$. Therefore, we can infer that the unobservable AP influences the processing of device diversity.

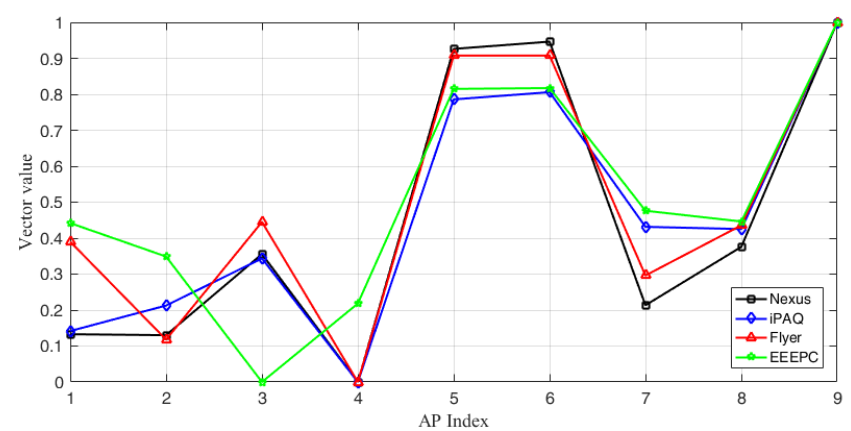

Figure 4. Comparison of the results of the new normalized RSS $\boldsymbol{\alpha}_{1}^{\prime}$ from different devices

Similarly, the SSD algorithm will inevitably take the unobservable AP as the reference AP and create the fingerprints by subtracting the RSS measurement of the reference AP from the RSS measurements of the other APs. Due to the limited coverage of the AP, no matter which AP is selected as the reference AP, there are always some RPs that are not covered by the reference AP. We regard this reference AP as the unobservable AP in "some RPs", which represents that for the reference AP no signal is recorded at a certain RP. Therefore, the SSD algorithm cannot avoid the influence of the unobservable AP on the processing of device diversity, and it will decrease the positioning accuracy. In Section 4 , we conduct experiments to show the influence of the unobservable AP as the reference AP on positioning accuracy.

Figure 5 shows the diagram of the indoor positioning algorithm based on the normalizing RSS, and the algorithm of the NRSSD is summarized as the pseudo-code in Algorithm 1.

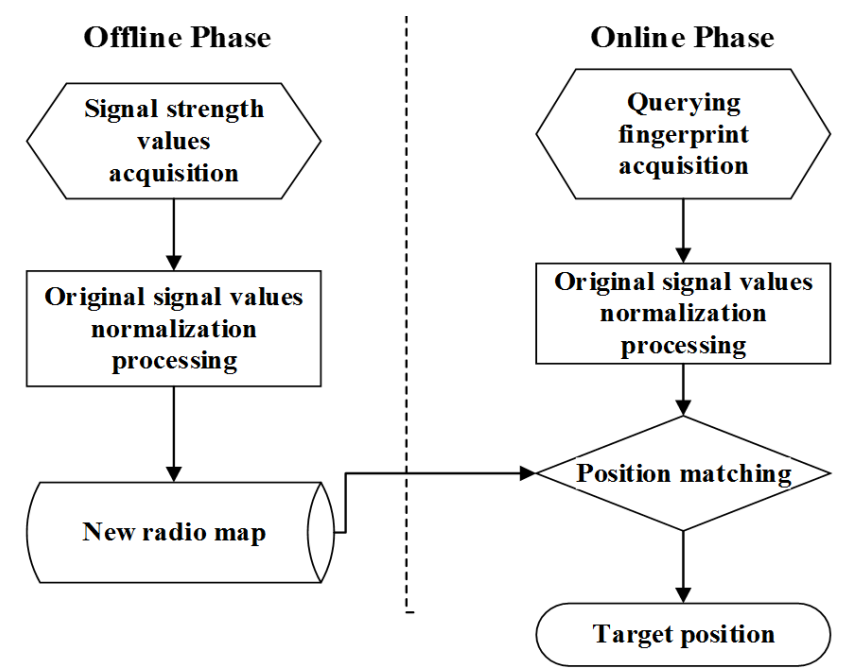

Figure 5. Diagram of the Indoor Positioning Algorithm based on the normalizing RSS 


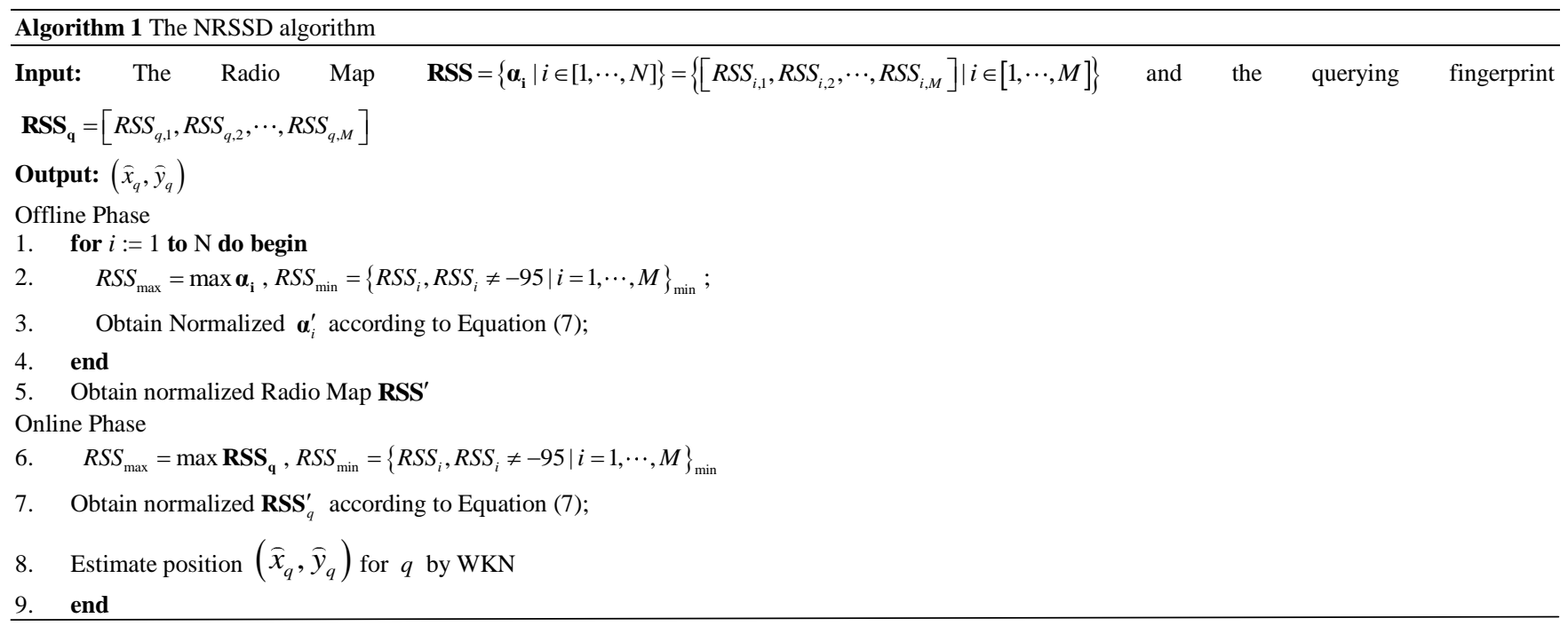

The dimension of the normalized fingerprints and the number of RPs dominate the running time of the NRSSD. The RPs number is $N$ and the APs number is $M$, which means that the dimension of $\boldsymbol{\alpha}^{\prime}$ is $M$. Compared with the DIFF and HLF, the NRSSD has the same number of RPs; however, it has fewer dimensions (the dimension of fingerprints is $\mathbf{C}_{M}^{2}$ in the DIFF and HLF). In addition, compared with the SSD, the NRSSD has more dimensions for fingerprints in theory; however, it can get a higher positioning accuracy (we will introduce it in Section 4). Therefore, the NRSSD has less running time compared with the DIFF and HLF.

\section{Experiment and Analysis}

\subsection{Datasets}

The experimental dataset is a released source dataset from the KIOS Research Center at the Department of Electrical and Computer Engineering of the University of Cyprus (the KIOS dataset) [10]. The Department of Electrical and Computer Engineering is a $560 \mathrm{~m}^{2}$ typical office environment. The researchers used four different mobile devices for data collection, namely, an HP IPAQ hw6915 PDA with Windows Mobile, an Asus EEEPC T101MT laptop running Windows 7, an HTC Flyer Android tablet, and an Android smart-phone (Samsung Nexus S). The dataset records the traces of signal strength of nine APs $(802.11 \mathrm{~b} / \mathrm{g})$ and contains the data from the offline phase and online phase. The offline fingerprints contain 2,100 measurements for 105 positions (20 each). The researchers collected additional querying fingerprints by walking along a predefined path. The path consists of 96 positions, most of which do not coincide with the reference points. Figure 6 is a diagram of the dataset's sampling from KIOS. The red points show the positions where querying fingerprints are collected, and the blue circles show the offline reference points.

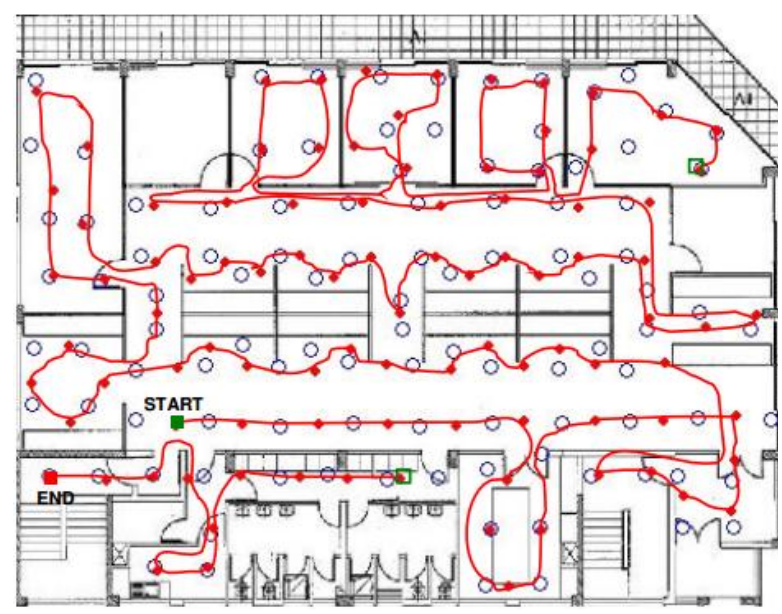

Figure 6. Diagram of the dataset's sampling from KIOS 


\subsection{Experimental Schemes}

The experiment is designed as follows:

\subsubsection{The Validity of Solving the Device Diversity Problem}

In this experiment, the NRSSD is compared with the traditional positioning algorithm using the original RSS. First, we normalize the original querying fingerprints from four devices as the inputs of the indoor fingerprinting positioning algorithm and use the normalized radio map from one device to locate the four kinds of devices. Then, we use one radio map from the original RSS from one device to locate the original querying fingerprints from the four kinds of devices. Finally, we compare the positioning errors of the two experiments.

\subsubsection{The Validity of the Positioning Accuracy}

In this experiment, the NRSSD is compared with the SSD, HLF, and DIFF. The training devices and testing devices are the same device (we use the Flyer device.) We use four methods to deal with the original RSS and acquire four kinds of fingerprint features. Four new radio maps consisting of new fingerprint features are used to estimate the respective positions. Finally, the average positioning errors of each algorithm are compared to verify the effectiveness of the NRSSD.

\subsubsection{The Validity of the Running Time}

In this experiment, we defined the running time as the average time in seconds that is required to obtain the precise position for one querying fingerprint. The running time plays a vital role in a successful indoor positioning system since less running time means a better user experience and less energy consumption for a user's mobile device.

\subsection{Results and Analysis}

\subsubsection{Experimental Results of Solving the Device Diversity Problem}

\subsubsection{Position Results using Original RSS}

For the Nexus, IPAQ, Flyer, and EEEPC devices, we use original RSS and normalized RSS selected from one device to locate the four kinds of devices. The average positioning errors are shown in Table 3.

Table 3. Results of verifying the validity on solving the device diversity problem

Table 3. Results of verifying the validity on solving the device diversity problem
\begin{tabular}{|c|c|c|c|c|c|c|}
\hline \multirow{2}{*}{ Average positioning errors (m) } & \multicolumn{5}{|c|}{ Training devices } \\
\cline { 3 - 7 } & & Nexus & IPAQ & Flyer & EEEPC \\
\hline \multirow{3}{*}{ Original RSS } & \multirow{3}{*}{$\begin{array}{c}\text { Testing } \\
\text { devices }\end{array}$} & IPAQ & 2.2752 & 7.1831 & 2.7189 & 2.9959 \\
\cline { 3 - 7 } & & Flyer & 2.2263 & 2.7124 & 5.8411 & 4.3880 \\
\cline { 3 - 7 } & & EEEPC & 2.8733 & 7.0854 & 1.9113 & 3.0309 \\
\hline \multirow{3}{*}{ NRSSD } & Nexus & 2.4686 & 2.5148 & 2.5509 & 2.5385 \\
\cline { 3 - 7 } & \multirow{3}{*}{$\begin{array}{c}\text { Testing } \\
\text { devices }\end{array}$} & IPAQ & 2.6135 & 2.3956 & 2.8105 & 2.7209 \\
\cline { 3 - 7 } & & Flyer & 2.6241 & 2.6589 & 2.2293 & 2.4298 \\
\cline { 3 - 7 } & EEEPC & 2.4286 & 2.7028 & 2.4449 & 2.4473 \\
\hline
\end{tabular}

Table 3 shows that the positioning accuracy of the four devices is obviously poor when using the original RSS. When using the radio map from the IPAQ device to locate other three devices, the average positioning errors are $7.1831 \mathrm{~m}$, $7.0854 \mathrm{~m}$, and $6.2458 \mathrm{~m}$, respectively, and to locate it, the average positioning error is only $2.7124 \mathrm{~m}$. For Flyer, when using the radio map (constructed by the Flyer device) to locate the other three devices, the positioning accuracy was reduced greatly compared to it and the growth rates of the average positioning errors are $42.25 \%, 205.61 \%$, and $59.91 \%$. The positioning errors' cumulative probability distribution functions (CDFs) for the two devices as training devices are shown in Figure 7.

\subsubsection{Positioning Results using the Normalized RSS}

For NRSSD, the positioning errors' CDFs for the IPAQ and Flyer devices being used as training devices are respectively shown in Figure 8. 


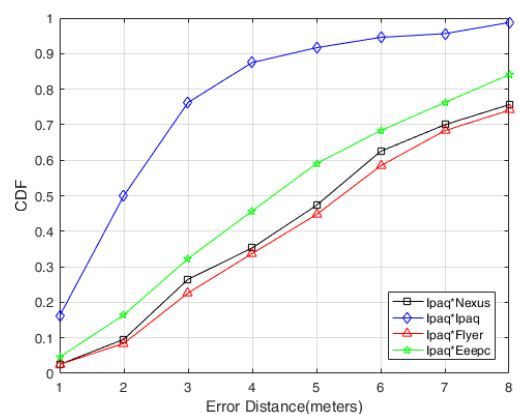

(a) IPAQ

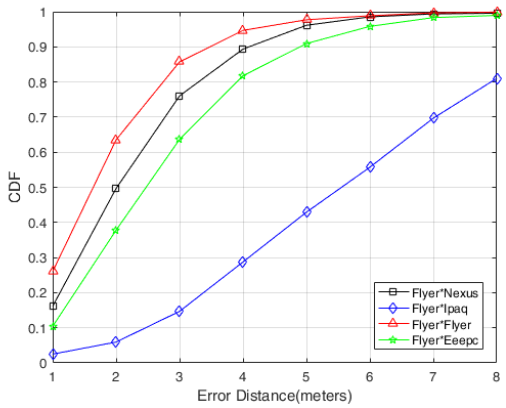

(b) Flyer

Figure 7. The CDFs of positioning errors for IPAQ and Flyer, respectively

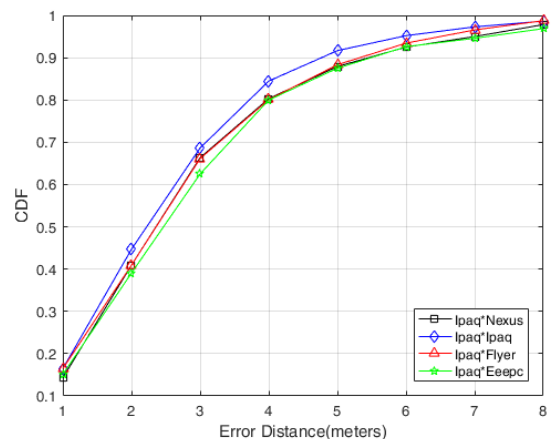

(a) IPAQ

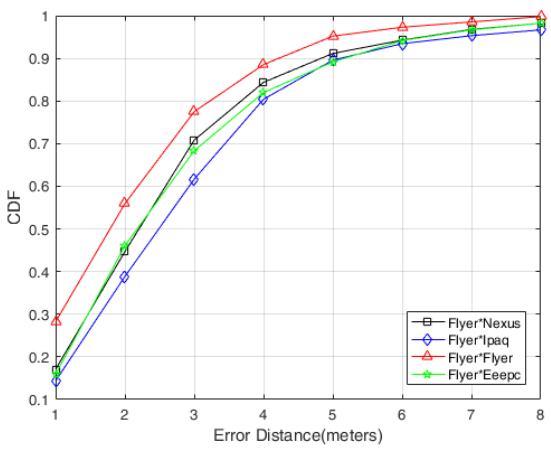

(b) Flyer

Figure 8. The CDFs of positioning errors with the normalized RSS for the IPAQ and Flyer, respectively

In Figure 8, we can see that large positioning errors caused by the device diversity have reduced significantly. Combined with the data in Table 4, when the radio map from the IPAQ with the normalized RSS is used to locate the other three testing devices, the average positioning errors are reduced from $7.1831 \mathrm{~m}$ to $2.5148 \mathrm{~m}, 7.0854 \mathrm{~m}$ to $2.6589 \mathrm{~m}$, and $6.2458 \mathrm{~m}$ to $2.7028 \mathrm{~m}$, and the average positioning errors are reduced $64.99 \%, 62.47 \%$, and $56.73 \%$.

Finally, from Table 4 and Figures 7 and 8, we can draw the following conclusion: the NRSSD can effectively solve the problem of device diversity, and one radio map can be applied to different devices, which clears the obstacle in the application of the indoor fingerprinting positioning technique.

Table 4. Contrast result of the average positioning errors for the four algorithms

\begin{tabular}{|c|c|c|c|c|}
\hline Algorithm & NRSSD & DIFF & SSD & HLF \\
\hline Positioning errors (m) & $\mathbf{2 . 2 2 9}$ & 2.441 & 2.557 & 2.627 \\
\hline
\end{tabular}

\subsubsection{Experimental Results of Positioning Accuracy}

This experiment mainly compares the positioning results of the NRSSD, DIFF, SSD, and HLF. We choose the Flyer device as the training device and testing device for this study. The CDFs of the positioning errors for the four algorithms are shown in Figure 9.

Figure 9 shows that the overall positioning accuracy of the proposed algorithm is superior to that of the other three algorithms. The NRSSD guarantees that $55.1 \%$ of the positioning errors are within $2 \mathrm{~m}$, which outperforms the DIFF, SSD, and HLF by $11.83 \%, 26.55 \%$, and $33.25 \%$, respectively.

To illustrate the influence of the unobservable AP on the SSD algorithm, we simulate two scenarios for comparison. The first scenario is the randomly selected reference AP in the SSD algorithm (after ten-fold cross-validation). The second scenario selects the weakest AP as the reference AP in the SSD algorithm. In the second scenario, the reference AP is not fixed, and we only guarantee that the weakest AP is selected at each RP, which ensures more unobservable APs are selected as the reference AP. We compare the CDFs of the positioning errors for two scenarios. The results are shown in Figure 10.

In Figure 10, compared with the randomly selected reference AP in the SSD, the positioning accuracy decreases when 
we select the weakest AP as the reference AP. Therefore, the selection of the reference AP influences the positioning accuracy, especially when the unobservable AP is the reference AP. In the NRSSD, we avoid the influence of the unobservable AP on device diversity processing and compare the CDFs of the positioning errors for the NRSSD and MM.

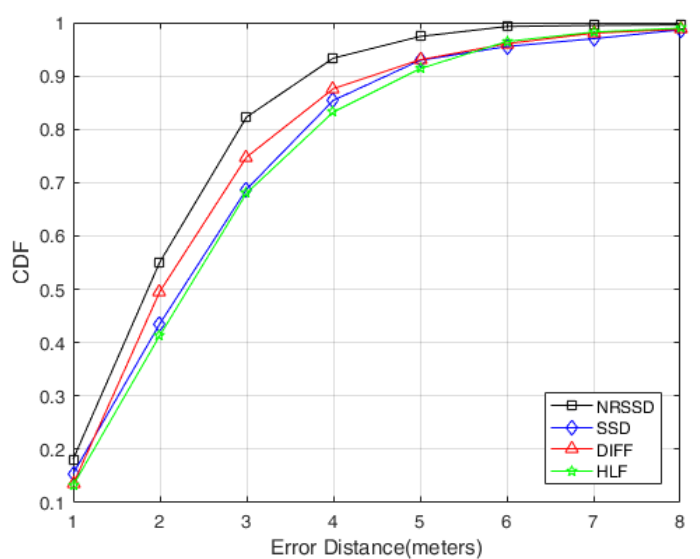

Figure 9. The CDFs of the positioning errors for four algorithms

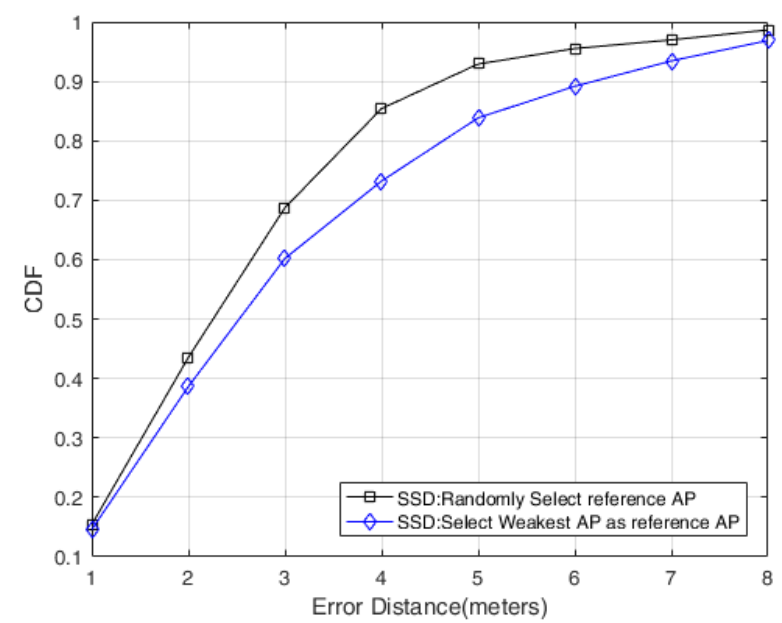

Figure 10. The CDFs of the positioning errors for the random selection reference AP and the weakest AP as the reference AP in the SSD algorithm

Figure 11 shows that the unobservable AP influences the processing of the device diversity, and the NRSSD performs well compared with the MM.

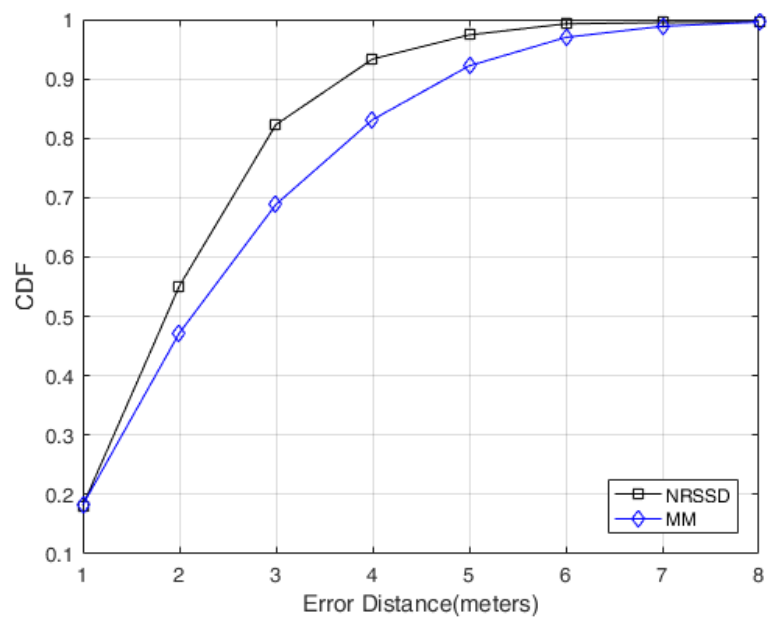

Figure 11. The CDFs of the positioning errors for the NRSSD and MM 
The average positioning errors and the running times of four the algorithms are compared, and the results are shown in Table 4 and Table 5, respectively.

As shown in Figure 9 and Tables 4 and 5, the average positioning error is 2.229m for the NRSSD, which is lower than that of the other three algorithms. Compared with the SSD, the NRSSD has no advantage in running time; however, it has an advantage in positioning accuracy. Compared with the DIFF and HLF, the NRSSD has an absolute advantage in terms of the running time and positioning errors. These findings prove that the NRSSD is more significant than the other algorithms.

Table 5. Contrast result of the running time using the four algorithms for one querying fingerprint

\begin{tabular}{|c|c|c|c|c|}
\hline Algorithm & NRSSD & DIFF & SSD & HLF \\
\hline Running time (ms) & $\mathbf{0 . 4 0 8 3}$ & 0.7305 & 0.1458 & 0.7381 \\
\hline
\end{tabular}

\section{Conclusions}

In order to solve the problem of device diversity, which hinders the application of the indoor positioning system, first, the paper understands the calculation method of signal values of the same AP received from different devices at the same position. We designed an indoor fingerprinting positioning algorithm based on the normalizing RSS to address device diversity. The basic advantage of our method is that it is more robust and stable than the original RSS, which can solve the RSS variance caused by device diversity. In addition, the proposed fingerprint feature using the normalized RSS has a much lower dimension than the fingerprint feature using the differential fingerprints. The experiment results show that the NRSSD of the overall positioning effect is superior to the SSD, DIFF, and HLF algorithms.

\section{Acknowledgements}

This paper is supported by the Key R\&D Plan of Shandong Province (NO.2018GGX101045), the National Key R\&D Plan (NO. 2018YFC0831002, 2017YFC0804406), the Key Project of Industrial Transformation and Upgrading (Made in China 2025 NO.TC170A5SW), Humanity and Social Science Fund of the Ministry of Education (NO.18YJAZH136, No. 17YJCZH262).

\section{References}

1. B. Buchli, "GPS-Equipped Wireless Sensor Network Node for High-Accuracy Positioning Applications," in Proceedings of the 9th European Conference on Wireless Sensor Networks, pp. 179-195, Trento, Italy, 2012

2. S. N. He and S. -H. G. Chan, "Wi-Fi Fingerprint-based Indoor Positioning: Recent Advances and Comparisons," IEEE Communications Surveys \& Tutorials, Vol. 18, No. 1, pp. 466-490, 2017

3. A. K. M. M. Hossain and W. S. Soh, "A Survey of Calibration-Free Indoor Positioning Systems," Computer Communications, Vol. 66, No. 15, pp. 1-13, July 2015

4. L. Z. Qiu and C. Liu, "An Improved Weighted K-Nearest Neighbor Algorithm for Indoor Positioning," Wireless Personal Communications, Vol. 96, No. 2, pp. 1-13, September 2017

5. A. W. Tsui, Y. H. Chuang, and H. H. Chu, "Unsupervised Learning for Solving RSS Hardware Variance Problem in WiFi Localization," Mobile Networks \& Applications, Vol. 14, No. 5, pp. 677-691, October 2009

6. H. Cheng, F. Wang, R. Tao, H. Luo, and F. Zhao, "Clustering Algorithms Research for Device-Clustering Localization," in Proceedings of International Conference on Indoor Positioning and Indoor Navigation IEEE, pp. 1-7, Sydney, NSW, Australia, 2012

7. J. G. Park, D. Curtis, S. Teller, and J. Ledlie, "Implications of Device Diversity for Organic Localization," in Proceedings of 2011 IEEE INFOCOM, pp. 3182-3190, Shanghai, China. April 2011

8. L. H. Chen, H. K. Wu, M. H. Jin, and G. H. Chen, "Homogeneous Features Utilization to Address the Device Heterogeneity Problem in Fingerprint Localization," IEEE Sensors Journal, Vol. 14, No. 4, pp. 998-1005, November 2014

9. C. Laoudias, D. Zeinalipour-Yazti, and C. G. Panayiotou, "Crowdsourced Indoor Localization for Diverse Devices Through Radiomap Fusion," in Proceedings of International Conference on Indoor Positioning and Indoor Navigation, pp. 1-7, Montbeliard-Belfort, France, 2013

10. C. Laoudias and C. G. Panayiotou, "Device Self-Calibration in Location Systems using Signal Strength Histograms," Journal of Location Based Services, Vol. 7, No. 3, pp. 165-181, September 2013

11. C. Figuera, "Time-Space Sampling and Mobile Device Calibration for WiFi Indoor Location Systems," IEEE Transactions on Mobile Computing, Vol. 10, No. 7, pp. 913-926, May 2011

12. A. Haeberlen, E. Flannery, A. M. Ladd, A. Rudys, D. S. Wallach, and L. E. Kavraki, "Practical Robust Localization over LargeScale 802.11 Wireless Networks," in Proceedings of the 10th Annual International Conference on Mobile Computing and Networking, pp. 70-84, 2004

13. F. Dong, Y. Chen, J. Liu, Q. Ning, and S. Piao, "A Calibration Free Localization Solution for Handling Signal Strength Variance," Mobile Entity Localization and Tracking in GPS less Environments, Springer Berlin Heidelberg, pp. 79-90, 2009

14. M. B. Kjærgaard, "Indoor Location Fingerprinting with Heterogeneous Clients," Pervasive and Mobile Computing, Vol. 7, No. 
1, pp. 31-43, February 2010

15. M. B. Kjærgaard, "Hyperbolic Location Fingerprinting: A Calibration-Free Solution for Handling Differences in Signal Strength," in Proceedings of 2008 Sixth Annual IEEE International Conference on Pervasive Computing and Communications (PerCom), pp. 110-116, Hong Kong, China, 2008

16. A. K. M. M. Hossain, Y. Jin, W. S. Soh, and H. N. Van, "SSD: A Robust RF Location Fingerprint Addressing Mobile Devices' Heterogeneity," IEEE Transactions on Mobile Computing, Vol. 12, No. 1, pp. 65-77, Jan. 2013

17. M. F. M. Mohsin, A. R. Hamdan, and A. A. Bakar, "The Effect of Normalization for Real Value Negative Selection Algorithm," in Proceedings of International Multi-Conference on Artificial Intelligence Technology, pp. 194-205, 2013

Chunxiu Li is a master's student in the School of Computer Science and Engineering at Shandong University of Science and Technology. Her research interests include machine learning and indoor localization.

Jianli Zhao received his Ph.D. in 2006 from the Department of Computer Application Technology at Northeastern University. In 2011, he served as an associate professor in the College of Computer Science and Engineering at Shandong University of Science and Technology. His current research interests include pervasive computing and indoor location.

Qiuxia Sun received her Ph.D. in system theory in 2011 from Qingdao University. She is currently an associate professor in the College of Mathematics and Systems Science at Shandong University of Science and Technology. Her research interests include big data analysis.

Xiang Gao is a master's student in the School of Computer Science and Engineering at Shandong University of Science and Technology. Her research interests include machine learning and indoor localization.

Guoqiang Sun is a master's student in the School of Computer Science and Engineering at Shandong University of Science and Technology. Her research interests include machine learning and indoor localization.

Chendi Zhu is a master's student in the School of Computer Science and Engineering at Shandong University of Science and Technology. Her research interests include machine learning and indoor localization. 